\title{
3d-Qsar Studies for Some Schiff Bases against Fungal Pathogen
}

\author{
Kishor Arora* \\ Department of Chemistry,Govt. P.G. College (Autonomous), Datia (M.P.) -475661, INDIA
}

\begin{abstract}
D$ QSAR viz. 3 descriptor quantitative structure and activity relationship studies for a series of compounds i.e. Schiff base derivatives using their semi-empirical quantum chemical descriptors and their laboratory reported activities against pathogen viz. . Colletotrichumfalcatum (ITCC 6656) have been carried out and reported in the present paper. Graphs between observed and predicted activities, both in the terms of $p(M I C) c a l$ and $p(M I C)$ obs. are also being reported on the basis of which this may be concluded that some parameters/descriptors have positive contribution towards the activity. The method has been proved to be useful tool for such type of studies.
\end{abstract}

Key words: Semiempirical/3D-qsar/fungal pathogen/Schiff bases compounds

\section{Introduction}

The battle against the infectious diseases has become a never ending process, as micro-organisms are becoming resistant more quickly than new drugs are being made available. Keeping this view, medicinal chemists are also trying out their best to speed up the drug discovery process for finding the lead molecule by using different techniques which includes QSAR (Quantitative Structure and Activity Relationship), CADD (Computer Aided Drug Design), Molecular Modeling, Combinatorial Chemistry and Microwave assisted synthesis. The use of these techniques has reduced the time required for primary screening of the molecule to locate the lead molecule.

Previously the studies involve the checking of antimicrobial activities of naturally occurring and /or synthesized compounds in the laboratory which may include organic compounds and inorganic complexes which were the areas of interest of many workers for so many years.

QSAR (Quantitative Structure and Activity Relationship) technique has been developed as a recent and finest technique in the field of drug designing. This technique has its foundation on the statistical analysis particular regression analysis of structural descriptors of compounds under studies with their lab. reported activities. In some of the recent references scientists have even utilized electronic properties or parameters of compounds/ drugs computed on the basis of Quantum Chemical Calculation viz. ab initio or semi-empirical studies as descriptors for QSAR studies and correlated these descriptors with activities of the compounds against a certain microorganism, which has been proved as a step ahead in the related field.

Schiff bases are the class of compounds in which an aldehyde and an amine condensed to form azomethinic linkage to yield a Schiff base. Schiff bases are of interest of many workers for so many years in the field of synthetic organic chemistry as well as in the synthesis and studies of complexes also. A lot of references including metal complexes with Schiff bases are known. Scientists are also trying to locate and study the antimicrobial activities of Schiff bases as well as of their metal complexes against various pathogens.

Literature survey carried out in the proposed field (1-32) revealed that on the workers/ scientists have tried many naturally occurring and synthetic compounds to check their activities against various microorganisms. QSAR studies of various series of compounds have also been carried out successfully. Some of the workers have also tried to correlate activities with 2 descriptors and 3 descriptors of the compounds pertaining to 2D- QSAR and 3D- QSAR studies(1-32).

Keeping above discussion in mind, in this present paper QSAR precisely 3D-QSAR studies which have been carried out on a new series of compounds i.e. Schiff base derivatives compounds shown in figure) with their activities against . Colletotrichumfalcatum (ITCC 6656) are reported.

\section{Preparation of Schiff bases:-}

\section{Experimental}

Schiff base compounds under study were synthesized by treating the appropriate amine $(\sim 1.0 \mathrm{mmol})$ with corresponding aldehyde $(\sim 1.1 \mathrm{mmol})$ in appropriate solvents such as ethyl alcohol and the reaction mixtures were refluxed for 6 to 12 hours using water condenser. In some cases products were obtained after cooling the reaction mixture as such while in some cases products were obtained after cooling it on ice bath. Schiff bases obtained were then filtered and re-crystallized using appropriate solvents i.e. either alcohol or ether (yield of the Schiff base compounds $\sim 60-70 \%$.) 
Compounds viz. Schiff bases (SB1-SB15) which are under study are shown in the figure -1. Analytical studies were also carried out for the synthesized compounds. These are given in the tables 1along with their melting points.

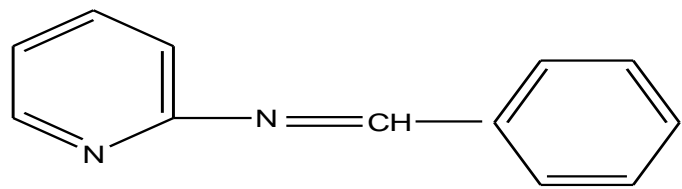

$2 \mathrm{~N}-$ [Benzalidene] Aminopyridine (SB-1)

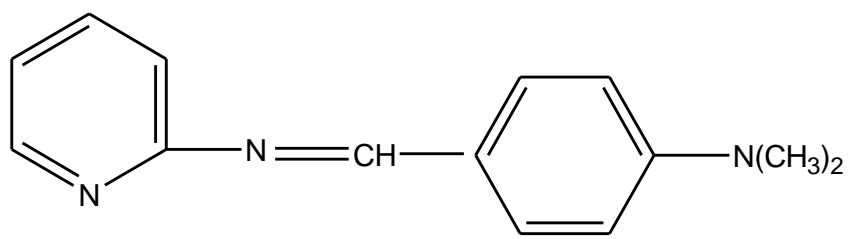

$2 \mathrm{~N}-$ [p-Dimethylaminobenzalidene] aminopyridine (SB-2)

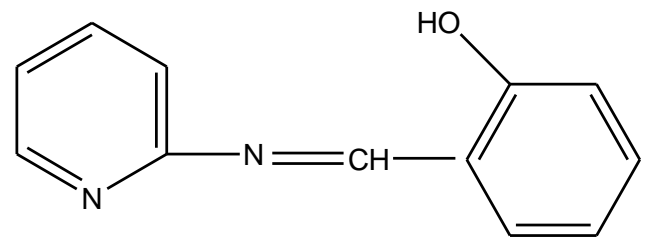

$2 \mathrm{~N}-[2-H y d r o x y b e n z a l i d e n e]$ aminopyridine (SB-3)

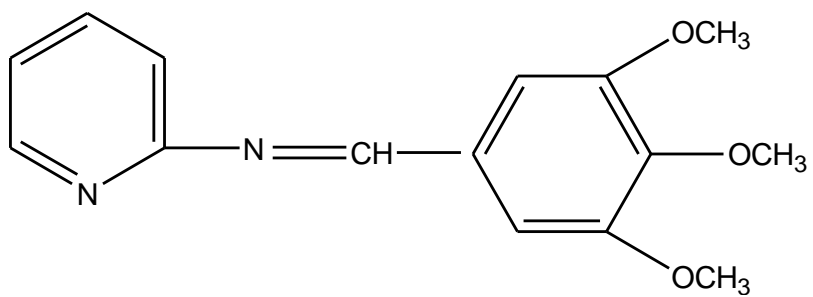

$2 \mathrm{~N}-[3,4,5$-Trimethoxybenzalidene $]$ aminopyridine (SB-4)

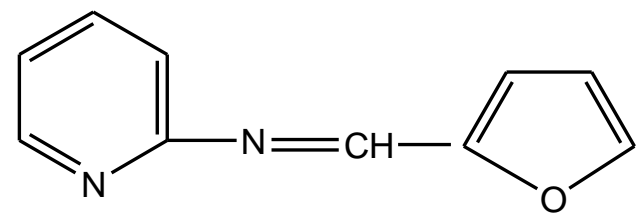

$2 \mathrm{~N}$ - [Furfuralidene] aminopyridine (SB-5)

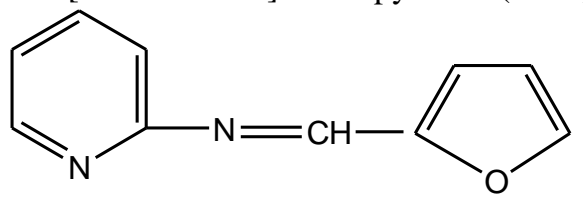

$3 \mathrm{~N}-$ [Furfuralidene] aminopyridine (SB-6)
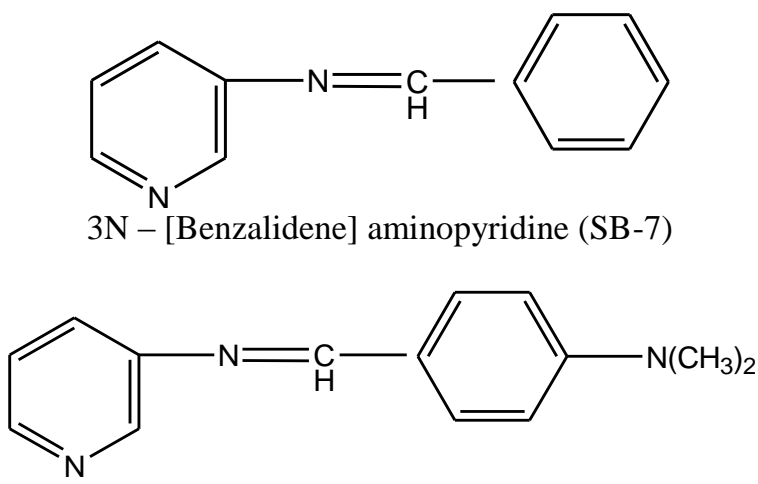

$3 \mathrm{~N}-[\mathrm{p}$-Dimethylaminobenzalidene] aminopyridine (SB-8) 


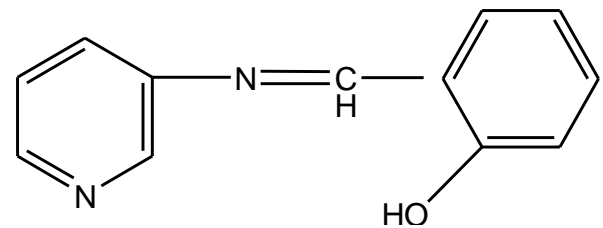

$3 \mathrm{~N}-[2-$ Hydroxybenzalidene] aminopyridine (SB-9)

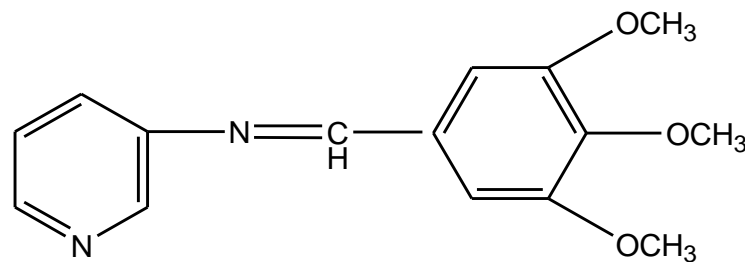

$3 \mathrm{~N}-[3,4,5$-Trimethoxybenzalidene] aminopyridine (SB-10)

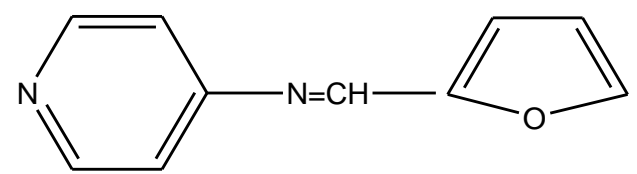

$4 \mathrm{~N}-$ [Furfuralidene] aminopyridine (SB-11)
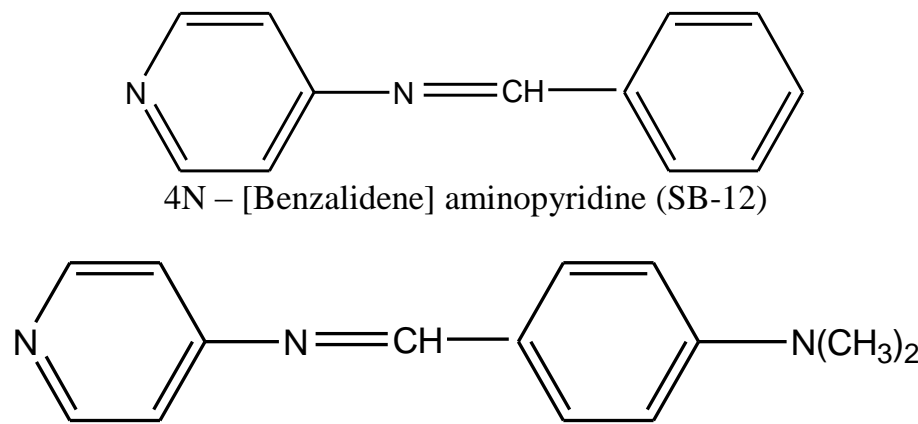

$4 \mathrm{~N}$ - [p-Dimethylaminobenzalidene] aminopyridine (SB-13)

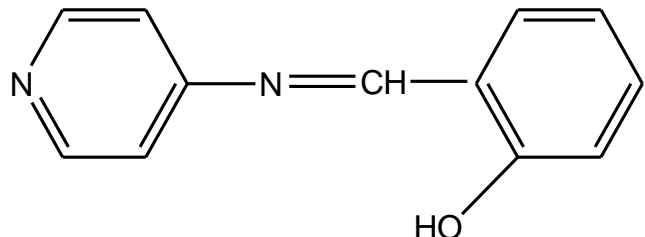

4N - [2-hydroxybenzalidene] aminopyridine (SB-14)

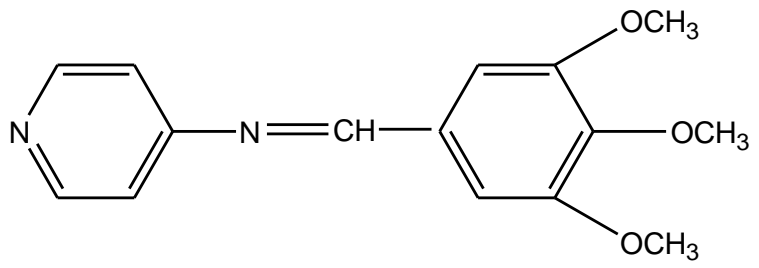

$4 \mathrm{~N}-[3,4,5$-Trimethoxy benzalidene] aminopyridine (SB-15)

Figure-1: Schiff Base Compounds Under Study 
Table 1: - Analytical studies for the Schiff bases under study

\begin{tabular}{|c|c|c|c|c|}
\hline Compound & $\begin{array}{c}\text { M. Pt. } \\
\left({ }^{0} \mathrm{C}\right)\end{array}$ & $\begin{array}{c}\text { C } \\
\text { Found } \\
\text { (Calcd.) }\end{array}$ & $\begin{array}{c}\text { H } \\
\text { Found } \\
\text { (Calcd.) }\end{array}$ & $\begin{array}{c}\mathrm{N} \\
\text { Found } \\
\text { (Calcd.) }\end{array}$ \\
\hline SB-1 & $180-185$ & $\begin{array}{c}76.26 \\
(79.3 \%)\end{array}$ & $\begin{array}{c}5.85 \\
(5.4 \%)\end{array}$ & $\begin{array}{c}18.28 \\
(15.3 \%)\end{array}$ \\
\hline SB-2 & $185-190$ & $\begin{array}{c}76.76 \\
(74.6 \%)\end{array}$ & $\begin{array}{c}5.67 \\
(6.66 \%)\end{array}$ & $\begin{array}{c}15.34 \\
(18.6 \%)\end{array}$ \\
\hline SB-3 & $190-192$ & $\begin{array}{c}70.9 \\
(72.0 \%)\end{array}$ & $\begin{array}{c}6.65 \\
(5.0 \%)\end{array}$ & $\begin{array}{c}13.05 \\
(14.0 \%)\end{array}$ \\
\hline SB-4 & $182-185$ & $\begin{array}{c}64.69 \\
(64.6 \%)\end{array}$ & $\begin{array}{c}6.004 \\
(6.1 \%)\end{array}$ & $\begin{array}{c}7.41 \\
(10.7 \%)\end{array}$ \\
\hline SB-5 & $168-172$ & $\begin{array}{c}67.46 \\
(69.7 \%)\end{array}$ & $\begin{array}{c}4.76 \\
(4.6 \%)\end{array}$ & $\begin{array}{c}14.86 \\
(16.2 \%)\end{array}$ \\
\hline SB-6 & $172-175$ & $\begin{array}{c}67.64 \\
(69.7 \%)\end{array}$ & $\begin{array}{c}4.67 \\
(4.6 \%)\end{array}$ & $\begin{array}{c}14.96 \\
(16.2 \%)\end{array}$ \\
\hline SB-7 & $182-189$ & $\begin{array}{c}77.62 \\
(79.3 \%)\end{array}$ & $\begin{array}{c}5.05 \\
(5.4 \%)\end{array}$ & $\begin{array}{c}17.82 \\
(15.3 \%)\end{array}$ \\
\hline SB-8 & $188-192$ & $\begin{array}{c}79.67 \\
(74.6 \%)\end{array}$ & $\begin{array}{c}7.67 \\
(6.66 \%)\end{array}$ & $\begin{array}{c}16.89 \\
(18.6 \%)\end{array}$ \\
\hline SB-9 & $175-180$ & $\begin{array}{c}70.91 \\
(72.0 \%)\end{array}$ & $\begin{array}{c}6.67 \\
(5.0 \%)\end{array}$ & $\begin{array}{c}13.50 \\
(14.0 \%)\end{array}$ \\
\hline SB-10 & $185-189$ & $\begin{array}{c}64.96 \\
(64.6 \%)\end{array}$ & $\begin{array}{c}6.40 \\
(6.1 \%)\end{array}$ & $\begin{array}{c}7.42 \\
(10.7 \%)\end{array}$ \\
\hline SB-11 & $170-174$ & $\begin{array}{c}64.56 \\
(69.7 \%)\end{array}$ & $\begin{array}{c}6.00 \\
(4.6 \%)\end{array}$ & $\begin{array}{c}14.20 \\
(16.2 \%)\end{array}$ \\
\hline SB-12 & 183-188 & $\begin{array}{c}76.82 \\
(79.3 \%)\end{array}$ & $\begin{array}{c}5.80 \\
(5.4 \%)\end{array}$ & $\begin{array}{c}18.80 \\
(15.3 \%)\end{array}$ \\
\hline SB-13 & $187-190$ & $\begin{array}{c}78.67 \\
(74.6 \%)\end{array}$ & $\begin{array}{c}7.77 \\
(6.66 \%)\end{array}$ & $\begin{array}{c}16.98 \\
(18.6 \%)\end{array}$ \\
\hline SB-14 & $176-181$ & $\begin{array}{c}70.9 \\
(72.0 \%)\end{array}$ & $\begin{array}{c}6.66 \\
(5.0 \%)\end{array}$ & $\begin{array}{c}12.4 \\
(14.0 \%)\end{array}$ \\
\hline SB-15 & 183-186 & $\begin{array}{c}66.08 \\
(64.6 \%)\end{array}$ & $\begin{array}{c}6.115 \\
(6.1 \%)\end{array}$ & $\begin{array}{c}7.813 \\
(10.7 \%)\end{array}$ \\
\hline
\end{tabular}

These compounds were also established on the basis of modern spectroscopic studies viz. i.r. spectral studies. N.M.R. spectral and Mass spectral studies for some of the representative compounds were also carried out.Analysis of the studies is given below:-

\section{I.R. spectral studies:-}

Assignments of peaks are given in the table-2 for Schiff base compounds. I.R spectral analysis show that $(\mathrm{C}=\mathrm{N})$ stretching azomethine peaks are predominant in the spectra of every Schiff base compound. This peak appears ca $1635-1590 \mathrm{~cm}^{-1}$ in the spectra of compounds under study. Apart from these peaks ring stretching peaks, $\mathrm{N}$ - phenyl stretching peak, ring breathing of benzene, $\mathrm{C}-\mathrm{N}-\mathrm{C}$ bending, $\mathrm{C}-\mathrm{H}$ out of plane deformation of mono substituted benzene and Out of plane bending of mono substituted benzene are also reported in the table-2.

\section{NMR spectral studies:-}

NMR spectral studies are of less interest for the compounds under study. However, these spectra were also recorded for some representative compounds on NMR spectrometer Bruker DRX-300 with due acknowledgement to C.D.R.I. Lucknow. Some notable peaks in these spectra are listed below:-

SB1: $1.062(\mathrm{~s}) ; 1.220$ (s); 2.118-2.065 (d); $2.490(\mathrm{~s}) ; 3.021(\mathrm{~s}) ; 3.341(\mathrm{~s}) ; 5.833(\mathrm{~s}) ; 6.161(\mathrm{~s}) ; 6.458-6.393(\mathrm{t})$; 6.584- 6.520 (t); 6.680-6.636(t); 7.227-7.206 (d); 7.302-7.278 (d); 7.376-7.353 (d); 7.464-7.736 (d); $7.534-$ $7.509(\mathrm{~d}) ; 7.665-7.585(\mathrm{q}) ; 7.828-7.687(\mathrm{q})$.

SB3:- 2.260 (s); 2.490 (s); 2.997 (s); 3.326 (s); 6.179-6.159 (d); 6.205(s0; 6.572-6.460 (d); 6.731- 6.599 (q); 6.925 (s); 6.991-6.971 (d); 7.100-7.073 (d);7.338-7.282 (t);7.463- 7.442 (d); 7.668-7.486( t); 7.919(s); 9.776 (s); 10.250(s); 10.695 (s) ppm respectively.

\section{MASS spectral studies:-}

Mass spectral studies of some representative Schiff base compounds have been carried out. Notable peaks in different compounds are:- base peaks at SB-1 :-228, SB-3 :-154, SB-4:- 273, SB-5: -173, with parent ion peaks at SB-1 :-181, SB-3 :-199, SB-4:- 273, SB-5: -173,. These parent peaks confirm the mol. weights of the compounds under study. Mass spectral analyses of the compounds are given in tables 3-6. 


\begin{tabular}{|c|c|c|c|c|c|c|c|c|}
\hline $\begin{array}{l}\text { Assigume } \\
\text { nt } \\
\text { Compoun } \\
\mathrm{d}\end{array}$ & $\begin{array}{c}(C=N) \\
\text { Stretching } \\
\text { azomethine }\end{array}$ & $\begin{array}{l}\text { King } \\
\text { stret- } \\
\text { ching }\end{array}$ & $\begin{array}{l}\text { N-Pheuly } \\
\text { stret-ching }\end{array}$ & $-N)$ & $\begin{array}{l}\text { Ring } \\
\text { breathing } \\
\text { of beuzene }\end{array}$ & $\begin{array}{l}\mathrm{C-N-} \\
\mathrm{C} \\
\text { Ben- } \\
\text { ding }\end{array}$ & $\begin{array}{l}\text { C-H out of plane } \\
\text { deformation of mono } \\
\text { substituted beuzene }\end{array}$ & $\begin{array}{l}\text { Out of plane } \\
\text { bending of } \\
\text { mono } \\
\text { substituted } \\
\text { beuzene }\end{array}$ \\
\hline SB-1 & 160015 & $\begin{array}{l}1515.8 \mathrm{~m} \\
1457.4 \mathrm{~s} \\
1434.4 \mathrm{~s}\end{array}$ & $\begin{array}{l}13965 \mathrm{~m} \\
1320.5 \mathrm{~m}\end{array}$ & $\begin{array}{l}1151 \\
2 \mathrm{~m}\end{array}$ & $\begin{array}{l}1088 \mathrm{~m} \\
988 \mathrm{~m}\end{array}$ & $\begin{array}{l}925 \mathrm{w} \\
857 \mathrm{w} \\
813 \mathrm{w}\end{array}$ & $\begin{array}{l}76 \mathrm{~m} \\
695 \mathrm{~m}\end{array}$ & $534 \mathrm{~m}$ \\
\hline $\mathrm{SB}-2$ & 15975 & $\begin{array}{l}1530 \mathrm{w} \\
1488 \mathrm{w} \\
1438 \mathrm{w}\end{array}$ & $\begin{array}{l}1370 \mathrm{~m} \\
1312 \mathrm{w}\end{array}$ & $\begin{array}{c}1165 \\
\mathrm{~m}\end{array}$ & $\begin{array}{l}1005 \mathrm{w} \\
991 \mathrm{w}\end{array}$ & $\begin{array}{l}95 \% \mathrm{w} \\
815 \mathrm{~m}\end{array}$ & $\begin{array}{l}725 \mathrm{w} \\
695 \mathrm{w}\end{array}$ & $511 \%$ \\
\hline SB-3 & 16085 & $\begin{array}{l}1552 \mathrm{~m} \\
1496 \mathrm{~m} \\
1428 \mathrm{~m}\end{array}$ & $\begin{array}{l}1400 \mathrm{w} \\
1353 \mathrm{w}\end{array}$ & $\underset{8}{1185}$ & $\begin{array}{l}1145 \mathrm{~m} \\
993 \mathrm{~m}\end{array}$ & $\begin{array}{l}913 \mathrm{~m} \\
845 \mathrm{~m}\end{array}$ & $\begin{array}{l}754 \mathrm{~m} \\
695 \mathrm{sh}\end{array}$ & $527 \pi$ \\
\hline $98 \cdot 4$ & 15915 & $\begin{array}{l}1555 \mathrm{sh} \\
1503 \mathrm{~m} \\
1421 \mathrm{~m}\end{array}$ & $\begin{array}{l}1391 \mathrm{~m} \\
1330 \mathrm{~s}\end{array}$ & $\begin{array}{c}1180 \\
5\end{array}$ & $\begin{array}{l}1127 \mathrm{~s} \\
989 \mathrm{~m}\end{array}$ & $915 \mathrm{w}$ & $677 \mathrm{w}$ & $525 \%$ \\
\hline SB-5 & 16005 & $\begin{array}{l}1569 \mathrm{w} \\
1482 \mathrm{~m} \\
1439 \mathrm{~m}\end{array}$ & $\begin{array}{l}1392 \mathrm{w} \\
1313 \mathrm{~m}\end{array}$ & $\underset{w}{1150}$ & $\begin{array}{l}1125 \mathrm{gh} \\
988 \mathrm{w}\end{array}$ & $925 \mathrm{w}$ & $695 \mathrm{sh}$ & $553 \pi$ \\
\hline SB-6 & $1624 \mathrm{~s}$ & $\begin{array}{l}1583 \mathrm{~s} \\
1525 \mathrm{~m} \\
1482 \mathrm{~s}\end{array}$ & $\begin{array}{l}1390 \mathrm{~h} \\
1293 \mathrm{~m}\end{array}$ & $\underset{w}{1186}$ & $\begin{array}{l}1131 \mathrm{w} \\
990 \mathrm{sh}\end{array}$ & $925 \mathrm{w}$ & $795 \mathrm{~m}$ & $550 \%$ \\
\hline SE-? & 16555 & $\begin{array}{l}1583 \mathrm{w} \\
1482 \mathrm{w} \\
1420 \mathrm{w}\end{array}$ & $1294 \mathrm{w}$ & $\begin{array}{c}1187 \\
\mathrm{~m}\end{array}$ & $1132 \mathrm{~m}$ & 8855 & $\begin{array}{l}798 \mathrm{~m} \\
706 \mathrm{~m}\end{array}$ & $550 \%$ \\
\hline SE-8 & 15998 & $\begin{array}{l}1551 \mathrm{~m} \\
1527 \mathrm{w} \\
1437 \mathrm{w}\end{array}$ & $\begin{array}{l}1398 \mathrm{~m} \\
1330 \mathrm{w}\end{array}$ & $\begin{array}{c}1169 \\
8\end{array}$ & $\begin{array}{l}1124 \mathrm{w} \\
1064 \mathrm{w}\end{array}$ & $942 \pi$ & $\begin{array}{l}817 \mathrm{~m} \\
708 \mathrm{~m}\end{array}$ & $516 \mathrm{~m}$ \\
\hline SB-9 & 15955 & $\begin{array}{l}1535 \mathrm{w} \\
1480 \mathrm{w} \\
1438 \mathrm{w}\end{array}$ & $\begin{array}{l}1355 \mathrm{~m} \\
1312 \mathrm{w}\end{array}$ & $\begin{array}{c}1160 \\
m\end{array}$ & $\begin{array}{l}1060 \mathrm{w} \\
991 \mathrm{w}\end{array}$ & $\begin{array}{l}955 \mathrm{~m} \\
815 \mathrm{~m}\end{array}$ & $\begin{array}{l}725 \mathrm{w} \\
695 \mathrm{w}\end{array}$ & $515 \pi$ \\
\hline SB-10 & 16245 & $\begin{array}{l}15855 \\
1539 \mathrm{~s} \\
1502 \mathrm{~s}\end{array}$ & $\begin{array}{l}1372 \mathrm{~m} \\
1329 \mathrm{~s}\end{array}$ & $\begin{array}{c}1185 \\
:\end{array}$ & $\begin{array}{l}71265 \\
1041 \mathrm{~s}\end{array}$ & $950 \%$ & $\begin{array}{l}842 \mathrm{w} \\
710 \mathrm{~m}\end{array}$ & $515 \mathrm{w}$ \\
\hline SE-11 & $1600 \mathrm{vg}$ & $\begin{array}{l}1585 \mathrm{sh} \\
1513 \mathrm{~m} \\
1423 \mathrm{~m}\end{array}$ & $\begin{array}{l}1372 \mathrm{sh} \\
1309 \mathrm{~m}\end{array}$ & $\begin{array}{c}1214 \\
8\end{array}$ & $\begin{array}{l}1129 \mathrm{~m} \\
1087 \mathrm{~m}\end{array}$ & $933 \mathrm{~m}$ & $\begin{array}{l}868 \mathrm{w} \\
816 \mathrm{~m}\end{array}$ & $531 \mathrm{~m}$ \\
\hline$\$ B \cdot 12$ & 16055 & $\begin{array}{l}1510 \mathrm{~m} \\
1455 \mathrm{~s} \\
1434 \mathrm{~s}\end{array}$ & $\begin{array}{l}1395 \mathrm{w} \\
1325 \mathrm{~m}\end{array}$ & $\begin{array}{c}1150 \\
\mathrm{~m}\end{array}$ & $\begin{array}{l}1088 \mathrm{~m} \\
988 \mathrm{~m}\end{array}$ & $\begin{array}{l}925 \mathrm{w} \\
857 \mathrm{w} \\
813 \mathrm{w}\end{array}$ & $\begin{array}{l}760 \mathrm{~m} \\
690 \mathrm{~m}\end{array}$ & $534 \mathrm{~m}$ \\
\hline SB-13 & $1600 \mathrm{~s}$ & $\begin{array}{l}1585 \mathrm{~m} \\
1512 \mathrm{~m}\end{array}$ & $\begin{array}{l}1431 \mathrm{w} \\
1331 \mathrm{w}\end{array}$ & $\begin{array}{c}1216 \\
\mathrm{w}\end{array}$ & $\begin{array}{l}1166 \% \\
1085 \mathrm{sh}\end{array}$ & $935 \mathrm{sh}$ & $\begin{array}{l}820 \mathrm{~m} \\
725 \mathrm{w}\end{array}$ & $534 \pi$ \\
\hline SB-14 & 16045 & $\begin{array}{l}1527 \mathrm{~s} \\
1510 \mathrm{sh}\end{array}$ & $\begin{array}{l}1478 \mathrm{~m} \\
1435 \mathrm{w} \\
1343 \mathrm{~m}\end{array}$ & $\underset{w}{1269}$ & $\begin{array}{l}119 \mathrm{~m} \\
1085 \mathrm{sh}\end{array}$ & 940 :h & $\begin{array}{l}828 \mathrm{~m} \\
760 \mathrm{~m}\end{array}$ & $525 \mathrm{w}$ \\
\hline SB-15 & $1590 \mathrm{vg}$ & $\begin{array}{r}1505 \mathrm{~s} \\
1461 \mathrm{~s} \\
1425 \mathrm{~m}\end{array}$ & $\begin{array}{l}1425 \mathrm{~m} \\
1391 \mathrm{~s} \\
1330 \mathrm{~s}\end{array}$ & $\begin{array}{c}1256 \\
\mathrm{~m}\end{array}$ & $\begin{array}{l}1188 \mathrm{~m} \\
1127 \mathrm{~s}\end{array}$ & $990 \mathrm{~m}$ & $\begin{array}{l}843 \mathrm{~m} \\
757 \mathrm{~m}\end{array}$ & $533 \mathrm{~m}$ \\
\hline
\end{tabular}

Table-3:-Mass spectral data for Schiff base (SB-1)

\begin{tabular}{|l|l|}
\hline $\mathbf{m} / \mathbf{e}$ & \%relative abundance \\
\hline 228 & 100 (Base peak) \\
\hline 227 & 10 \\
\hline 212 & 15 \\
\hline 197 & 15 \\
\hline 189 & 18 \\
\hline 185 & 18 \\
\hline 181 & 18 (Parent peak) \\
\hline 155 & 35 \\
\hline 154 & 75 \\
\hline 144 & 25 \\
\hline 136 & 65 \\
\hline 122 & 45 \\
\hline 110 & 62 \\
\hline 98 & 60 \\
\hline 97 & 15 \\
\hline 95 & 100 \\
\hline
\end{tabular}

Table-4:-Mass spectral data for Schiff base (SB-3)

\begin{tabular}{|l|l|}
\hline $\mathbf{m} / \mathbf{e}$ & \%relative abundance \\
\hline 199 & 10 (parent peak) \\
\hline 189 & 05 \\
\hline 164 & 10 \\
\hline 155 & 28 \\
\hline 154 & 100 (base peak) \\
\hline 150 & 40 \\
\hline 138 & 30 \\
\hline 137 & 52 \\
\hline 136 & 70 \\
\hline 107 & 22 \\
\hline 95 & 15 \\
\hline
\end{tabular}


Table -5:-Mass spectral data for Schiff base (SB-4)

\begin{tabular}{|l|l|}
\hline $\mathbf{m} / \mathbf{e}$ & \%relative abundance \\
\hline 173 & 10 \\
\hline 197 & 10 \\
\hline 219 & 10 \\
\hline 273 & 100 (base peak) \\
\hline 274 & 20 \\
\hline 295 & 02 \\
\hline 305 & 15 \\
\hline 323 & 05 \\
\hline 415 & 04 \\
\hline 420 & 02 \\
\hline 441 & 02 \\
\hline
\end{tabular}

Table -6:-Mass spectral data for Schiff base (SB-5)

\begin{tabular}{|l|l|}
\hline $\mathbf{m} / \mathbf{e}$ & \%relative abundance \\
\hline 173 & 100 (base peak) \\
\hline 174 & 20 \\
\hline 189 & 04 \\
\hline 195 & 10 \\
\hline 205 & 03 \\
\hline 211 & 04 \\
\hline 227 & 04 \\
\hline 278 & 03 \\
\hline
\end{tabular}

Antimicrobial activities of the compounds under study:-

Procurement of pathogens:-ITCC certified Pathogens viz. Colletotrichumfalcatum (ITCC 6656) were procured from plant pathology division, I.A.R.I., New Delhi with due acknowledgement. Their sub- cultures have been made and the anti-microbial studies of the compounds under study were carried out against these pathogens at Birla Institute of Professional Studies with due acknowledgements. MIC (minimum inhibitory concentration) have been recorded which is then subjected to further studies to get QSAR equations. Experimental procedures that have been adopted for anti microbial studies are mentioned below:

\section{Experimental procedure for Anti fungal studies:-}

Pure culture of Colletotrichumfalcatum obtained from I.A.R.I. was revived. Fresh inoculation of the fungal species was done by taking a loopful from I.A.R.I. culture tube and streaking was done on sterile PDA plates and slants and broth culture in sterile PDB incubated at $\sim 24^{0} \mathrm{c}$ for $5-7$ days to revive the culture. After revival of culture petri plates were prepared and for this purpose PDA was used. Media were cooled and plates were prepared by dispensing $15-20 \mathrm{~mL}$ media per plate. Plates were kept inverted in the incubator at $\sim 24^{0} \mathrm{c}$ overnight for sterility checking. Paper discs were made from whatmann filter paper using paper piercing instrument with a diameter of $\sim 6 \mathrm{~mm}$ and sterilized by keeping them in an incubator at $\sim 70^{\circ} \mathrm{c}$ for 24 hrs. About $100 \mu \mathrm{L}$ of actively growing pathogen from the culture was used to inoculate sterile PDA plates through spread plate method. The filter paper discs were dispensed on glass plates and each was loaded with $5 \mu \mathrm{L}$ volume of pre decided dilution. These discs were left air dried in the laminar air flow and then carefully transferred to inoculated plates at pre designated positions. The plates were then inoculated at $\sim 24^{0} \mathrm{c}$ for $5-7$ days.

Table-7:-Antifungal analysis of Schiff base compounds under study against Colletotrichumfalcatum (ITCC NO- 6656)

\begin{tabular}{|c|c|c|c|c|}
\hline \multirow{2}{*}{$\begin{array}{l}\text { Compounds under } \\
\text { study }\end{array}$} & \multicolumn{4}{|c|}{ Concentrations Of compounds $/ 10 \mathrm{ml}$} \\
\hline & $10 \mathrm{mg}$ & $20 \mathrm{mg}$ & $30 \mathrm{mg}$ & $40 \mathrm{mg}$ \\
\hline SB1 & $\begin{array}{ll}------ \\
\end{array}$ & --------- & ------- & ----- \\
\hline SB2 & $\begin{array}{l}------- \\
\end{array}$ & ---------- & ------- & ------- \\
\hline SB3 & -------- & ----------- & $\begin{array}{ll}------ \\
\end{array}$ & $10 \mathrm{~mm}$ \\
\hline SB4 & -------- & ----------- & $11 \mathrm{~mm}$ & $13 \mathrm{~mm}$ \\
\hline SB5 & -------- & ---------- & -------- & -------- \\
\hline SB6 & --------- & ---------- & -------- & $\begin{array}{ll}------- \\
\end{array}$ \\
\hline SB7 & --------- & --------- & --------- & $15 \mathrm{~mm}$ \\
\hline SB8 & -------- & --------- & --------- & $10 \mathrm{~mm}$ \\
\hline SB9 & --------- & ---------- & ----------- & ------- \\
\hline SB10 & |--------- & $20 \mathrm{~mm}$ & $29 \mathrm{~mm}$ & $30 \mathrm{~mm}$ \\
\hline SB11 & |--------- & ---------- & ----------- & $10 \mathrm{~mm}$ \\
\hline SB12 & -------- & --------- & $\begin{array}{l}------- \\
\end{array}$ & $10 \mathrm{~mm}$ \\
\hline SB13 & -------- & --------- & $18 \mathrm{~mm}$ & $20 \mathrm{~mm}$ \\
\hline SB14 & |------- & ---------- & ---------- & ------- \\
\hline SB15 & $10 \mathrm{~mm}$ & $12 \mathrm{~mm}$ & $13 \mathrm{~mm}$ & $18 \mathrm{~mm}$ \\
\hline
\end{tabular}




\section{Computational Details for the computational work:-}

The AM1Hamiltonia on Hyperchem 8.0 professional version were used to calculate the QSAR related descriptors such as Hydration energy (Hyd E), $\log$ P (log P), Refractivity (REF), Polarizability (POL). mass (mass), Surface area approx (SAA), Surface area Grid (SAG), volume (Vol), Heat of formation (HF), Zero point energy (ZPE), HOMO energy (HOMO), LUMO energy ( LUMO) and dipole moment ( DM).

All these computations were carried out on Pentium core -2 duo machine having configuration Intel $(\mathrm{R})$ core $^{(\mathrm{TM})} 2$ Duo CPU

$\mathrm{T}_{5450} @ 1.66 \mathrm{GHz}$.

\section{$982 \mathrm{MHz}, 896 \mathrm{MB}$ RAM}

\section{GB HDD}

with windows - Microsoft windows XP software as an operating system.

These descriptors and the activities of the compounds against specified microbes, mentioned in the tables, were taken into consideration in terms of $\mathrm{p}$ (MIC) and were subjected to statistical analysis/ regression analysis to get QSAR equations. All these statistical calculations were carried out on the same machine mentioned above using EXCEL software.

\section{Results and Discussion}

The in-vitro anti microbial viz. anti bacterial and anti fungal studies of the Schiff base compounds were carried out as per experimental procedure mentioned in the experimental section at Birla Institute of Professional Studies, Gwalior with due acknowledgement. The activities of the compounds were recorded in terms of Minimum Inhibitory Concentration (MIC). Some of the compounds have shown notable activities against the specified pathogen under study while other do not show any activity. The activities are recorded and reported here in the table 7 . Figures of some of the plates were also taken showing growth of the pathogens..

These labs.reported activities of the Schiff base compounds under study in terms of $\mathrm{p}(\mathrm{MIC})$ have been subjected to QSAR studies with Semi-empirical quantum chemical based certain parameters of the compounds. These parameters/ descriptors are hydration energy $(\mathrm{HE}), \log \mathrm{P}(\log \mathrm{P})$, Refractivity(RF), Polarizaility (POL), Mass(Mass), Surface area approx(SAA), Surface area Grid( SAG), Volume (VOL), Heat of formation (HF), Zero point Energy (ZPE), HOMO energy(HOMO), LUMO energy (LUMO) and dipole moment( DM).

These parameters are computed on HYPERCHEM 8.0 version software after geometry optimization of the compounds under study as mentioned in the experimental section above. The computed parameters/ descriptors are reported in the tables 8-11.

The methods adopted here in this project are AM1, PM3, MNDO and ZINDO respectively. All methods are proved to be good to give optimized geometry and minimized energy for the compounds under study. This fact can also be proved as IR studies which are observed experimentally for the compounds under study match well with the computed IR spectral frequencies using Hyperchem 8.0 professional software especially on the basis of AM1 and PM3 method. The fact of authenticity of AM1 and PM3 may prove to be appropriate in this study, so far as software is concerned.

The software Hyperchem 8.0 version was quite fast and provides reasonably good results regarding all parameters viz. physical parameters, spectroscopic parameters and SAR related parameters.

Table-8 : Semi- Empirical AM1 based Computed properties of the Compounds (SB1-SB15) under study and their antifungal results $\mathrm{p}(\mathrm{MIC})$ on Colletotrichumfalcatum (ITCC NO- 6656)

\begin{tabular}{|c|c|c|c|c|c|c|c|}
\hline Compd & SAA & SAG & HE & Log P & RF & POL & Mass \\
\hline SB-1 & 328.43 & 403.81 & -5.27 & 1.7 & 63.44 & 22.15 & 182 \\
\hline SB-2 & 431.84 & 474.32 & -4.13 & 0.75 & 77.06 & 27.17 & 225.29 \\
\hline SB-3 & 331.54 & 407.3 & -10.03 & 0.67 & 65.05 & 22.79 & 198.22 \\
\hline SB-4 & 316.5 & 431.39 & -5.44 & -1.28 & 82.56 & 29.57 & 272.3 \\
\hline SB-5 & 307.6 & 373.6 & -8.83 & -0.13 & 53.02 & 19.31 & 172.19 \\
\hline SB-6 & 220.04 & 393.91 & 13.97 & -0.31 & 58.09 & 21.34 & 188.23 \\
\hline SB-7 & 321.31 & 387.75 & -5.91 & 0.13 & 64.08 & 22.15 & 182.22 \\
\hline SB-8 & 419.03 & 458.97 & -4.74 & -0.82 & 77.69 & 27.17 & 225.29 \\
\hline SB-9 & 511.03 & 396.31 & 25.91 & -0.89 & 65.68 & 22.79 & 198.22 \\
\hline SB-10 & 410.85 & 489.58 & -7.99 & -2.85 & 83.2 & 29.57 & 272.3 \\
\hline SB-11 & 300.68 & 362.1 & -9.65 & -1.82 & 53.58 & 19.31 & 172.19 \\
\hline SB-12 & 320.5 & 387.2 & -6.12 & 0.01 & 64 & 22.15 & 182.22 \\
\hline SB-13 & 418.19 & 459.23 & -4.95 & -0.94 & -7.62 & 27.17 & 225.29 \\
\hline SB-14 & 191.46 & 382.67 & -2.87 & -1.02 & 65.61 & 22.79 & 198.22 \\
\hline SB-15 & 386.88 & 482.8 & -8.37 & -2.97 & 83.12 & 29.57 & 272.3 \\
\hline
\end{tabular}


3d-Qsar Studies for Some Schiff Bases against Fungal Pathogen

\begin{tabular}{|c|c|c|c|c|c|c|c|}
\hline VOL & HF & ZPE & HOMO & LUMO & DM & MIC & $\begin{array}{c}\text { P(MIC) } \\
\text { obs. }\end{array}$ \\
\hline 625.06 & 90.6906 & 122.856 & -0.6919 & 0.28678 & 0.846 & 40 & -1.6021 \\
\hline 756.97 & 93.4313 & 168.516 & -0.4682 & 0.46352 & 2.391 & 40 & -1.6021 \\
\hline 637.89 & 41.6617 & 125.925 & -0.6392 & 0.31279 & 1.173 & 40 & -1.6021 \\
\hline 708.65 & 93.8518 & 183.267 & -0.0233 & 0.78834 & 10.492 & 30 & -1.4771 \\
\hline 565.99 & 64.5137 & 296.708 & -0.5966 & 0.31456 & 0.518 & 40 & -1.6021 \\
\hline 624.46 & 83.3848 & 129.024 & -1.0503 & 0.41409 & 1.739 & 40 & -1.6021 \\
\hline 603.63 & 93.2951 & 122.589 & -0.8216 & 0.224 & 1.532 & 40 & -1.6021 \\
\hline 737.98 & 101.265 & 168.037 & -0.5746 & 0.41979 & 3.794 & 40 & -1.6021 \\
\hline 636.32 & 72.8485 & 123.336 & -0.9824 & 0.01622 & 0.04 & 40 & -1.6021 \\
\hline 850.52 & 24.9715 & 185.623 & -0.0456 & 0.04697 & 3.247 & 20 & -1.301 \\
\hline 549.61 & 70.8844 & 103.81 & -0.7422 & 0.21055 & 3.084 & 40 & -1.6021 \\
\hline 604.65 & 93.9724 & 122.609 & -0.8569 & 0.15012 & 3.463 & 40 & -1.6021 \\
\hline 736.8 & 101.813 & 168.075 & -0.6006 & 0.36356 & 6.029 & 30 & -1.4771 \\
\hline 606.8 & 115.656 & 123.955 & -0.1917 & 0.22419 & 3.773 & 40 & -1.6021 \\
\hline 813.78 & 25.1947 & 185.703 & -0.5742 & 0.07244 & 2.504 & 10 & -1 \\
\hline
\end{tabular}

Table -9: Semi- Empirical PM3 based Computed properties of the Compounds (SB1-SB15) under study and their antifungal results p(MIC) on Colletotrichumfalcatum (ITCC NO- 6656)

\begin{tabular}{|c|c|c|c|c|c|c|c|}
\hline Compd & MIC & P(MIC)Obs. & SAA & SAG & HE & Log P & RF \\
\hline SB-1 & 10 & -1.6021 & 327.9 & 398.57 & -5.21 & 1.7 & 63.44 \\
\hline SB-2 & 40 & -1.6021 & 429.81 & 471.29 & -4.09 & 0.75 & 77.06 \\
\hline SB-3 & 40 & -1.6021 & 330.69 & 405.7 & -10.08 & 0.67 & 65.05 \\
\hline SB-4 & 40 & -1.4771 & 421.13 & 487.55 & -6.75 & -1.28 & 82.56 \\
\hline SB-5 & 40 & -1.6021 & 308.07 & 373.46 & -8.7 & -0.13 & 53.02 \\
\hline SB-6 & 10 & -1.6021 & 240.24 & 396.48 & 15.86 & -0.31 & 58.09 \\
\hline SB-7 & 10 & -1.6021 & 321.22 & 386.16 & -5.91 & 0.13 & 64.08 \\
\hline SB-8 & 40 & -1.6021 & 407.33 & 448.7 & -4.5 & -0.82 & 77.69 \\
\hline SB-9 & 40 & -1.6021 & 806.97 & 394.77 & 31.12 & -0.89 & 65.58 \\
\hline SB-10 & 40 & -1.301 & 346.15 & 448.96 & -6.48 & -2.85 & 83.2 \\
\hline SB-11 & 40 & -1.6021 & 300.33 & 358.64 & -9.57 & -1.82 & 53.58 \\
\hline SB-12 & 30 & -1.6021 & 320.33 & 386.98 & -6.11 & 0.01 & 64 \\
\hline SB-13 & 30 & -1.4771 & 300.14 & 463.82 & -3.11 & -0.94 & 77.62 \\
\hline SB-14 & 40 & -1.6021 & -637.25 & 399.25 & 21.37 & -1.02 & 65.61 \\
\hline SB-15 & 40 & -1 & 438.28 & 494.15 & -8.31 & -2.97 & 83.12 \\
\hline
\end{tabular}

\begin{tabular}{|c|c|c|c|c|c|c|c|}
\hline POL & Mass & VOL & HF & ZPE & HOMO & LUMO & DM \\
\hline 22.15 & 182.22 & 617.65 & 74.5392 & 118.0805 & -0.0237 & 0.26053 & 0.856 \\
\hline 27.17 & 225.29 & 751.72 & 75.0653 & 161.0597 & -0.6047 & 0.12665 & 1.636 \\
\hline 22.79 & 198.22 & 635.43 & 31.13328 & 121.3319 & -0.7363 & 0.07505 & 0.576 \\
\hline 2.57 & 272.3 & 808.85 & 23.0389 & 177.9144 & -0.1781 & 0.2533 & 3.178 \\
\hline 19.31 & 172.19 & 565.62 & 45.417 & 298.6673 & -0.6761 & 0.01641 & 0.0695 \\
\hline 21.34 & 188.23 & 626.51 & 67.5881 & 123.476 & -1.1512 & 0.26996 & 1.601 \\
\hline 22.15 & 152.22 & 601.46 & 84.0827 & 117.0909 & -0.908 & 0.000038 & 1.271 \\
\hline 27.17 & 225.29 & 733.56 & 73.9653 & 163.41169 & -0.4841 & 0.05898 & 2.219 \\
\hline 22.79 & 198.22 & 637.16 & 69.1811 & 118.1698 & -0.0854 & 0.18165 & 0.219 \\
\hline 29.57 & 272.3 & 761.35 & 20.0201 & 180.9994 & -0.2461 & 0.06722 & 3.985 \\
\hline 19.31 & 172.19 & 547.72 & 53.6628 & 99.6895 & -0.8176 & 0.05231 & 3.093 \\
\hline 22.15 & 182.22 & 601.83 & 84.5714 & 17.8388 & -0.9709 & 0.005465 & 3.068 \\
\hline 27.17 & 237.3 & 764.27 & 19.88857 & 166.303 & -0.2295 & 0.19077 & 4.018 \\
\hline 22.79 & 198.22 & 639.93 & 82.8752 & 118.0187 & -0.1425 & 0.1323 & 2.432 \\
\hline 29.57 & 272.3 & 818.5 & 17.18738 & 177.1228 & -0.0148 & 0.10017 & 3.332 \\
\hline
\end{tabular}

Table-10: Semi- Empirical MNDO based Computed properties of the Compounds (SB1-SB15) under study and their antifungal results p(MIC) on Colletotrichumfalcatum (ITCC NO- 6656)

\begin{tabular}{|c|c|c|c|c|c|c|c|}
\hline Compd & MIC & P(MIC)Obs. & SAA & SAG & HE & Log P & RF \\
\hline SB-1 & 40 & -1.6021 & 330.44 & 398.47 & -5.25 & 1.7 & 63.44 \\
\hline SB-2 & 40 & -1.6021 & 431.87 & 474.32 & -4.13 & 0.75 & 77.06 \\
\hline SB-3 & 40 & -1.6021 & 334.34 & 409.26 & -10.21 & 0.67 & 65.05 \\
\hline SB-4 & 30 & -1.4771 & 390.26 & 501.86 & -7.57 & -1.28 & 82.56 \\
\hline SB-5 & 40 & -1.6021 & 308.07 & 372.46 & -8.7 & -0.13 & 53.02 \\
\hline SB-6 & 40 & -1.6021 & 363.32 & 421.19 & 28.06 & -0.31 & 58.09 \\
\hline SB-7 & 40 & -1.6021 & 325.42 & 390.74 & -6 & 0.13 & 64.05 \\
\hline SB-8 & 40 & -1.6021 & 424.74 & 461.48 & -4.85 & -0.82 & 77.69 \\
\hline SB-9 & 40 & -1.6021 & 335.53 & 410.08 & 11.66 & -0.84 & 65.69 \\
\hline SB-10 & 20 & -1.301 & 441.86 & 516.88 & -8.45 & -2.85 & 83.2 \\
\hline SB-11 & 40 & -1.6021 & 516.88 & 364.47 & -9.63 & -1.82 & 53.58 \\
\hline SB-12 & 40 & -1.6021 & 324.51 & 390.82 & -6.13 & 0.01 & 64 \\
\hline
\end{tabular}


3d-Qsar Studies for Some Schiff Bases against Fungal Pathogen

\begin{tabular}{|c|c|c|c|c|c|c|c|}
\hline SB-13 & 30 & -1.4771 & 424.08 & 462.98 & -5.04 & -0.94 & 77.62 \\
\hline SB-14 & 40 & -1.6021 & 85.62 & 385.41 & -2.18 & -1.02 & 65.61 \\
\hline SB-15 & 10 & -1 & 373.02 & 493.92 & -8.61 & -2.97 & 83.12 \\
\hline POL & Mass & VOL & HF & ZPE & HOMO & LUMO & DM \\
\hline 22.15 & 182.22 & 620.52 & 72.6814 & 125.5213 & -0.7304 & 0.1807 & 0.115 \\
\hline 27.17 & 225.29 & 756.97 & 83.6483 & 173.1405 & -0.552 & 0.3057 & 1.985 \\
\hline 22.79 & 198.22 & 639.44 & 26.4478 & 128.8318 & -0.6656 & 0.164 & 1.313 \\
\hline 29.57 & 272.3 & 831.31 & 86.8148 & 187.9244 & -0.2584 & 0.7191 & 9.788 \\
\hline 19.31 & 172.19 & 565.62 & 37.5895 & 294.6581 & -0.5852 & 0.2013 & 0.256 \\
\hline 21.34 & 188.23 & 653.43 & 77.1433 & 132.8215 & -1.0426 & 0.2484 & 1.872 \\
\hline 22.15 & 182.22 & 607.45 & 90.8727 & 125.2745 & -0.8436 & 0.0489 & 1.666 \\
\hline 27.17 & 225.29 & 744.34 & $1-1.5124$ & 172.8898 & -0.6482 & 0.2094 & 3.487 \\
\hline 22.79 & 198.22 & 653.5 & 76.8839 & 126.1077 & -0.111 & 0.0129 & 0.94 \\
\hline 29.57 & 271.3 & 851.71 & 39.9456 & 190.328 & -0.1264 & 0.0924 & 2.72 \\
\hline 19.31 & 172.19 & 552.73 & 54.2342 & 106.3981 & -0.7498 & 0.1216 & 3.434 \\
\hline 22.15 & 182.22 & 608.03 & 99.2275 & 125.2744 & -0.0444 & 0.07092 & 3.738 \\
\hline 27.17 & 225.29 & 745.39 & 101.729 & 172.9119 & -0.7083 & 0.12267 & 5.8 \\
\hline 22.79 & 198.22 & 611.87 & 118.4008 & 127.213 & -0.3373 & 0.06016 & 3.922 \\
\hline 29.57 & 272.3 & 829.41 & 67.5956 & 189.1327 & -0.911 & 0.7773 & 9.452 \\
\hline
\end{tabular}

Table -11: Semi- Empirical ZINDO based Computed properties of the Compounds (SB1-SB15) under study and their antifungal results $\mathrm{P}(\mathrm{MIC})$ on Colletotrichumfalcatum (ITCC NO- 6656)

\begin{tabular}{|c|c|c|c|c|c|c|c|}
\hline Compd & MIC & P(MIC)Obs. & SAA & SAG & HE & Log P & RF \\
\hline SB-1 & 40 & -1.6021 & 326.31 & 398.74 & -5.13 & 1.7 & 63.44 \\
\hline SB-2 & 40 & -1.6021 & 424.59 & 468.15 & -3.98 & 0.75 & 77.06 \\
\hline SB-3 & 40 & -1.6021 & 328.99 & 410.75 & -9.8 & 0.67 & 65.05 \\
\hline SB-4 & 30 & -1.4771 & 302.76 & 441.39 & -7.29 & -1.29 & 82.56 \\
\hline SB-5 & 40 & -1.6021 & 304.93 & 369.03 & -8.63 & -0.013 & 53.02 \\
\hline SB-6 & 40 & -1.6021 & -181.81 & 374.77 & -8.95 & -0.31 & 58.09 \\
\hline SB-7 & 40 & -1.6021 & 319.51 & 385.78 & -5.81 & 0.13 & 64.08 \\
\hline SB-8 & 40 & -1.6021 & 417.14 & 454.99 & -4.72 & -0.82 & 77.69 \\
\hline SB-9 & 40 & -1.6021 & 208.81 & 396.28 & -7.19 & -0.89 & 65.68 \\
\hline SB-10 & 20 & -1.301 & 369.07 & 456.56 & -7.15 & -2.85 & 83.2 \\
\hline SB-11 & 40 & -1.6021 & 296.07 & 355.04 & -9.52 & -1.82 & 53.08 \\
\hline SB-12 & 40 & -1.6021 & 318.2 & 381.79 & -6.09 & 0.01 & 64 \\
\hline SB-13 & 30 & -1.4771 & 415.76 & 455.86 & -4.93 & -0.94 & 77.62 \\
\hline SB-14 & 40 & -1.6021 & -515.87 & 371.28 & 557.06 & -1.02 & 65.61 \\
\hline SB-15 & 10 & -1 & 320.41 & 440.74 & -7.49 & -2.49 & 83.12 \\
\hline
\end{tabular}

\begin{tabular}{|c|c|c|c|c|c|c|c|}
\hline POL & Mass & VOL & HF & ZPE & HOMO & LUMO & DM \\
\hline 22.15 & 183.22 & 614.22 & -5357.263 & 166.065 & -7.266 & 5.3892 & 0.767 \\
\hline 27.17 & 225.29 & 744.51 & -6733.5149 & 229.561 & -5.7525 & 5.6997 & 1.445 \\
\hline 22.29 & 198.22 & 636.3 & -5499.3121 & 169.919 & -7.177 & 5.8793 & 2.46 \\
\hline 29.57 & 272.3 & 735.94 & -7446.8667 & 255.6673 & -4.8535 & 5.0977 & 3.882 \\
\hline 19.31 & 172.19 & 561.53 & -4598.1684 & 328.6815 & -6.9848 & 5.6676 & 1.415 \\
\hline 21.34 & 188.23 & 584.88 & -5392.7584 & 180.5573 & -5.7055 & 4.316 & 1.846 \\
\hline 22.15 & 182.22 & 599.51 & -5342.8922 & 166.099 & -6.8912 & 5.4596 & 1.358 \\
\hline 27.17 & 225.29 & 729.78 & -6719.1172 & 229.5434 & -5.7808 & 5.7748 & 3.079 \\
\hline 22.79 & 198.22 & 620.61 & -5502.9938 & 170.1358 & -6.8308 & 5.0378 & 7.069 \\
\hline 29.57 & 272.3 & 770.33 & -7388.4733 & 252.558 & -6.6451 & 5.8622 & 2.089 \\
\hline 19.31 & 172.19 & 542.8 & -4583.9114 & 140.7262 & -7.2156 & 5.4445 & 3.347 \\
\hline 22.15 & 182.22 & 599.36 & -5344.7538 & 166.1238 & -7.2369 & 5.1767 & 3.849 \\
\hline 27.17 & 225.29 & 728.99 & -6721.2763 & 229.5709 & -5.9184 & 5.4844 & 5.915 \\
\hline 22.79 & 198.22 & 590.34 & -5460.0367 & 169.2597 & -3.8549 & 6.7071 & 4.554 \\
\hline 29.17 & 272.3 & 741.15 & -7519.3218 & 253.8556 & -4.1665 & 6.5701 & 7.667 \\
\hline
\end{tabular}

This final equation was used to get predicted activities for all the series of compounds and against all microorganisms under study. The final 3D QSAR equations are reported as under:-

AM1/Schiff base compounds (SB1-SB15)/AntifungalColletotrichumfalcatum (ITCC NO- 6656) /3Dequation

$\mathrm{P}(\mathrm{MIC})=-0.058595368(\log \mathrm{P})+0.000791347($ Mass $)+0.000632045(\mathrm{VOL})-2.154266796$

$\mathrm{N}=15, \mathrm{SD}=0.104560234, \mathrm{r}=0.834310522, \mathrm{~F}$ test $=8.397675409$

PM3/Schiff base compounds (SB1-SB15)/AntifungalColletotrichumfalcatum (ITCC NO- 6656) /3Dequation 
$\mathrm{P}(\mathrm{MIC})=-0.05113144(\log \mathrm{P})+0.001480447($ Mass $)-0.001375507(\mathrm{HF})-1.793867934$

$\mathrm{N}=15, \mathrm{SD}=0.107689734, \mathrm{r}=0.823169914, \mathrm{~F}$ test $=7.706676044$

MNDO/Schiff base compounds (SB1-SB15)/AntifungalColletotrichumfalcatum （ITCC NO- 6656) /3Dequation

$\mathrm{P}(\mathrm{MIC})=-0.037797391(\log \mathrm{P})+0.003834886($ Mass $)-0.000507095(\mathrm{VOL})-2.01860159$

$\mathrm{N}=15, \mathrm{SD}=0.107080072, \mathrm{r}=0.825377898, \mathrm{~F}$ test $=7.836553464$

ZINDO/Schiff base compounds (SB1-SB15)/AntifungalColletotrichumfalcatum (ITCC NO- 6656) /3Dequation

$\mathrm{P}(\mathrm{MIC})=-0.037797391(\log \mathrm{P})+0.0041666($ Mass $)+5.2409 \mathrm{E}-05(\mathrm{HF})-2.11514388$

$\mathrm{N}=15, \mathrm{SD}=0.112498247, \mathrm{r}=0.805093409, \mathrm{~F}$ test $=6.755193075$

Graphs were also drawn between observed activities and predicted activities, both in the terms of $\mathrm{p}$ (MIC) form 3 D-QSAR equations for this series of compounds. These trend line graphs are shown in figure 3-6.

\section{Conclusions}

This method has once again proved to be useful for this type of studies. The parameters/ descriptors which contribute positively to $\mathrm{p}$ (MIC) in final 3D QSAR equations are listed below:-

AM1/ Schiff bases (SB1-SB15)/ Colletotrichumfalcatum (ITCC NO- 6656): - Log P, Mass and VOL PM3/ Schiff bases (SB1-SB15)/ Colletotrichumfalcatum (ITCC NO- 6656): - Log P, Mass and HF MNDO/ Schiff bases (SB1-SB15)/ Colletotrichumfalcatum (ITCC NO- 6656): Log P, HE and VOL ZINDO/ Schiff bases (SB1-SB15)/ Colletotrichumfalcatum (ITCC NO- 6656): Log P, DM and HF

In conclusion this may be said that these parameters / descriptors have more impact on (MIC) over all other descriptors computed and reported.

\section{Acknowledgements}

Author sincerely acknowledges M.P.Council of Science and Technology, Bhopal for financial assistance inthe form of Research Project sanctioned to him. \{Endt. No. 6051/CST/R\&D/2011 Dated 31-03$2011\}$

Figure -3 Trend line Graph between p(MIC) observed and AM1 computed p(MIC) calculated for the Schiff base compounds (SB1-SB15)under study for their antifungal activities on Colletotrichumfalcatum (ITCC NO- 6656)

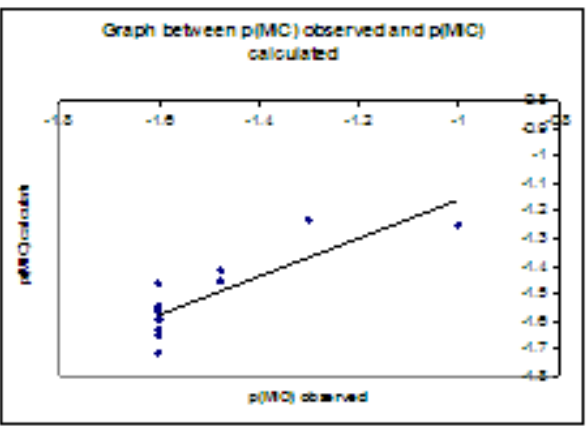

\begin{tabular}{|c|c|}
\hline $\mathrm{p}(\mathrm{MIC})$ obsered & $\mathrm{p}(\mathrm{MIC})$ calculated \\
\hline-1.602 & -1.715 \\
\hline-1.602 & -1.541 \\
\hline-1.602 & -1.633 \\
\hline-1.477 & -1.416 \\
\hline-1.602 & -1.653 \\
\hline-1.602 & -1.592 \\
\hline-1.602 & -1.636 \\
\hline-1.602 & -1.461 \\
\hline-1.602 & -1.543 \\
\hline-1.301 & -1.234 \\
\hline-1.602 & -1.564 \\
\hline-1.602 & -1.628 \\
\hline-1.477 & -1.455 \\
\hline-1.602 & -1.554 \\
\hline-1 & -1.25 \\
\hline
\end{tabular}


Figure -4 Trend line Graph between p(MIC) observed and PM3 computed p(MIC) calculated for the Schiff base compounds (SB1-SB15)under study for their antifungal activities on Colletotrichumfalcatum (ITCC NO- 6656)

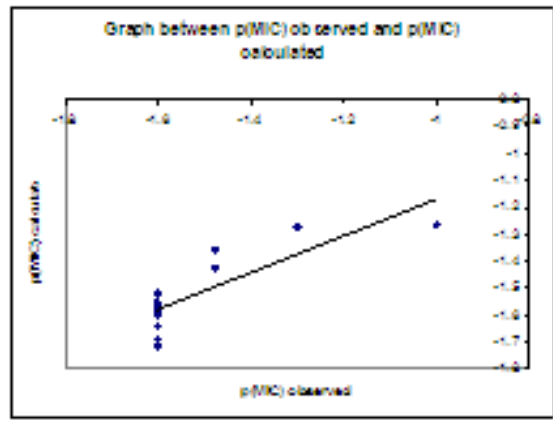

\begin{tabular}{|c|c|}
\hline P(MIC) (Observed) & P(MIC) (Calculated) \\
\hline-1.602 & -1.714 \\
\hline-1.602 & -1.602 \\
\hline-1.602 & -1.577 \\
\hline-1.477 & -1.357 \\
\hline-1.602 & -1.595 \\
\hline-1.602 & -1.592 \\
\hline-1.602 & -1.691 \\
\hline-1.602 & -1.52 \\
\hline-1.602 & -1.55 \\
\hline-1.301 & -1.273 \\
\hline-1.602 & -1.52 \\
\hline-1.602 & -1.641 \\
\hline-1.477 & -1.422 \\
\hline-1.602 & -1.562 \\
\hline-1 & -1.263 \\
\hline & \\
\hline & \\
\hline
\end{tabular}

Figure-5 Trend line Graph between $p$ (MIC) observed and MNDO computed $p$ (MIC) calculated for the Schiff base compounds (SB1-SB15)under study for their antifungal activities onColletotrichumfalcatum (ITCC NO- 6656)

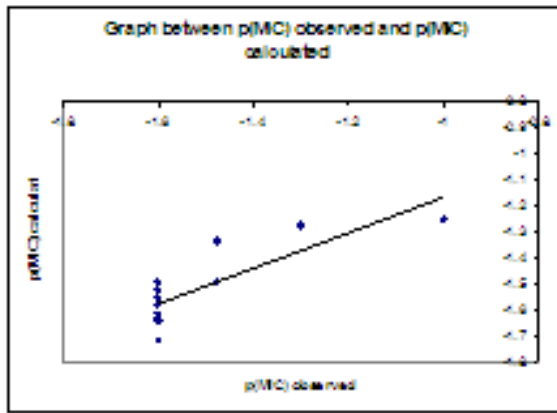

\begin{tabular}{|c|c|}
\hline $\mathrm{p}(\mathrm{MIC})$ observed & $\mathrm{p}(\mathrm{MIC})$ calculated \\
\hline-1.602 & -1.717 \\
\hline-1.602 & -1.575 \\
\hline-1.602 & -1.615 \\
\hline-1.477 & -1.334 \\
\hline-1.602 & -1.639 \\
\hline-1.602 & -1.613 \\
\hline-1.602 & -1.634 \\
\hline-1.602 & -1.492 \\
\hline-1.602 & -1.549 \\
\hline-1.301 & -1.272 \\
\hline-1.602 & -1.551 \\
\hline-1.602 & -1.629 \\
\hline-1.477 & -1.487 \\
\hline-1.602 & -1.519 \\
\hline-1 & -1.251 \\
\hline
\end{tabular}


Figure -6 Trend line Graph between p(MIC) observed and ZINDO computed p(MIC) calculated for the Schiff base compounds (SB1-SB15)under study for their antifungal activities onColletotrichumfalcatum (ITCC NO- 6656)

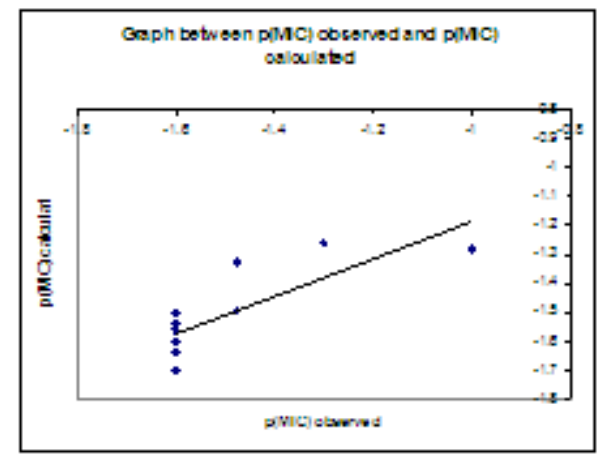

\begin{tabular}{|c|c|}
\hline $\mathrm{p}(\mathrm{MIC})$ observed & $\mathrm{p}(\mathrm{MIC})$ calculated \\
\hline-1.602 & -1.697 \\
\hline-1.602 & -1.558 \\
\hline-1.602 & -1.603 \\
\hline-1.477 & -1.322 \\
\hline-1.602 & -1.638 \\
\hline-1.602 & -1.602 \\
\hline-1.602 & -1.641 \\
\hline-1.602 & -1.498 \\
\hline-1.602 & -1.544 \\
\hline-1.301 & -1.26 \\
\hline-1.602 & -1.569 \\
\hline-1.602 & -1.636 \\
\hline-1.477 & -1.493 \\
\hline-1.602 & -1.537 \\
\hline-1 & -1.281 \\
\hline
\end{tabular}

References

[1]. "Epidemiological typing of Bacillus sp.isolated from food", HediSchraf, Marina Steele, Bruce MaNab, Josef Odummaru and Mansel W. Griffiths, Application and Environmental microbiology,62(11), 4229-4232, (1996).

[2]. "Characterization of post antifungal effect: comparative studies with amphotericin B , 5-fluoro cytosine and micorazole on candida albicans" Scalarone GM, Mikami Y, Kurita N.,Ichihana Y, Yazawz K and Miyaji M, Mycosex, 34, $297-302$ (1991).

[3]. "A synthetic HIV -I Rev inhibitor interfering with the CRM1- mediated nuclear export" Dirk Daelemans, Elena Afonina Jacob Nilson, Grudrunn Werner, Jorgen Kjems, Eric De Clercq, George N. Parlakis and AmneMiekeVandamme, Proceedings of National Academy of sciences of United States of America, 99(22), 14440-14445, (2002).

[4]. "Slide culture of tubercle Bacilli II in-vitro sensitivity testing” R.W. Read, Canadian J. of Microbiology, 1(1), 3035 (1955).

[5]. "Activity of different antibacterial per Erwiniaamylovora growth and cercropi", FabienneMourgues, Marie-Noelle Brisset and Elisabeth C.H., Plant Science, 139(1), 83-91,(1998).

[6]. "Postantibiotic and sub MIC effect on benzyl penicillin against streptococcus pnenmniiee with different susceptibilities for penicillin" IgnaOdenholt, Ingegerd, Gustafsson and Elisabeth Lowdin, Chemotherapy, 49,287-293, (2003).

[7]. "Synthesis and antifungal activity of novel 5-substituted-6- fluoro-4- alkyl(aryl) thioquinazoline derivatives" Xu GF, Song BA, Bhadury PS, Yang S. Zhang PQ, Jin LH, Xue w, Hu DY, Lu P, Bio org med . Chem. 15(11), 3768-74 (2007).

[8]. "Synthesis and fungicidal activity of tubulin polymerization promoters, part - I: pyrido[2,3-b]pyrazines" Crowley PJ, Lamberth C, Muller U, Wendeborn S, Nobel K, Williams J, Sageot OA, Carter N, Mathie T, Kempf HJ, Godwin J, Schneiter P, Dobler MR, Pest Manag. Sci, 66(2), 178-185 (2010).

[9]. "Studies on high coortdination complexes of dioxouranium (VI) with a Schiff base", KishorArora and K.P. Sharma, Synth. And React. In Inorg. And Met.-Org. Chem..32, 913 (2002).

[10]. "Synthesis and QSAR studies of O,O- diaryl-ethyl phosphorothionates for their fungicidal activities against RhiZoctoniasolani and Sclerotiumrolfsii”, Lakshman BA, Gupta RL, Indian J. of Chemistry, 44(B), 152-157, (2005).

[11]. "Antimicrobial activity of methanol extract of Origanwnmajorana L. ( Sweet marjoram) “, Leeja L, Thoopil JE, Journal of Environmental Biology, 28(1), 145-146 (2007).

[12]. "Chiral gamma- aryl- $1 \mathrm{H}-1,2,4$ triazolo derivatives as highly potential antifungal agents: Design, synthesis, structure and in-vitro fungicidal activities" CaO X, Li F, Hu M, Lu W, Yu GA, Liu SH, Agric. Food Chem., 56(23),11367-75 (2008).

[13]. "Synthesis fungicidal and insecticidal activities of beta- Methoxy acrylate containing N-Acetyl pyrazoline derivatives", Zhao PL, Wang F, Zhang MZ, Liu ZM, Huang W, Yang GF, , J Agric. Food Chem., 56(22), 10767-73 (2008).

[14]. "Synthesis and Fungicidal activity against Rhizoctoniasoleni of 2- Alkyl (Alkylthio)- 5- Pyrazolyl-1,3,4-oxadiazoles (Thiadiazoles)", HansongChen,ZhengmingLi and Yufeng Han, J. Agirc. Food Chem., 48(11), 5312-5315, (2000).

[15]. "Antimicrobial activity of amino acid, imidazole and sulphonamide derivatives of pyrazole [3,4-d] pyrimidines", Ghorab MM, Zeinab H Ismail, Soad M Abdel Gawad and Anhar Abdel Aziem, Heteroatom chem.., 15(1), 57-62, (2004).

[16]. "Synthesis and biological activities of novel diheterocyclic compounds containing1,2,4- triazolo-[1,5-a] pyrimidine and 1,3,4Oxadiazole", Zwning Liu, Guangfu Yang and Xianghua Qin, J. Chem. Technol. Biotechnol, 76, 1154-1158, (2001). 
[17]. "Design synthesis and in- vitro antibacterial/antifungal evaluation of novel 1-ethyl,-6- fluoro-1,4-dihydro-4-oxo-7-(1- piperazinyl) quinoline-3- carboxylic acid derivatives", Yu Z, Shi G, Sun Q, Jin H, Teng Y, Tao K, Zhou G, Liu W, Wen F, Hou T, Eur. J. of Med. Chem., 44(11), 4726-33, (2009).

[18]. "Coordination modes of a Schiff base pentadentate derivatives of 4-Amino antipyrine with Cobalt (II), Nickel (II) and Copper (II) metal ions: Synthesis, spectroscopic and antimicrobial studies", Chandra S, Jain D, Sharma AK, Sharma P, Molecules, 14(1), 174-90 ,(2009).

[19]. "AM1 study on the conformation of 6- aminopencillanic acid" BojjaRajeshwarRao, Indian J. Chem., 41(B), 1697-1701, (2002)

[20]. "Modeling chemical reactivity in Diels Alder cycloaddition reaction of heteroacyl-1,4- benzoquinones - A semiempirical (AM1) computational study", Pradassani RT, Paradosani P, Agarwal MM, Mathur G, Indian J Chem., 40(B), 518-521,(2001).

[21]. "QSAR analysis of analogs of bis [ 2- (acylamino)phenyl] disulfides, 2-(acylamino)benzenethiols and S-[2-(acylamino)phenyl] alkanethionates as anti-hyperlipidemic agents", Hanumanthrao P, Sambasivrao SV, Soni LK, Gupta AK, Kaskhedikar SG, Indian J. Chem., 44(B), 1481-1486,(2005).

[22]. "In-vitro antifungal studies of novel synthetic compounds against Alterneriabrassicae", Vinod Kr. Sewariya, RichaSrivastava, GBKS Prasad and KishorArora, Biosciences and Biotechnology Asia, 8(1),231-239 (2011).

[23]. "In-vitro evaluation of novel synthetic compounds against Alternariabrassicicola", Vinod Kr. Sewariya, RichaSrivastava, GBKS Prasad and KishorArora, Research journal of Pharmaceutical biological and Chemical sciences, 3(3), 360, (2012).

[24]. “In-vitro evaluation of novel synthetic compounds for control of black rot disease of XanthomonasCampasteris", Vinod Kr. Sewariya, RichaSrivastava, GBKS Prasad and KishorArora, International Journal of Pharma and Biosciences, 3(1),B, 441-453, (2012).

[25]. "Invitro evaluation of novel synthetic compounds againstFusariumSacchari", Vinod Kr. Sewariya, RichaSrivastava, GBKS Prasad and KishorArora, International Journal of Pharma and Biosciences,3(3)B, 910-920, (2012).

[26]. "In-vitro antimicrobial studies of some pyrazolones and their SAR studies" KishorArora and VeenaNathani, Asian J. Chem., 24(12),5803-5805, (2012).

[27]. "Anti-microbial and QSAR studies of some pyrazolones compounds", KishorArora and VeenaNathani, Research journal of Pharmaceutical biological and Chemical sciences, 3(4), 1423, (2012).

[28]. "QSAR studies of some pyrazolones as antimicrobial agent", KishorArora and VeenaNathani, International Journal of Pharma and Biosciences, 4(1), 657-671, (2013).

[29]. "Semi-empirical based 3D-QSAR studies of some pharmacological important compounds", International journal of Pharma and Bopsciences, KishorArora, 4(2), 244, (2013).

[30]. "Synthesis spectral and microbial studies of some novel Schiff base derivatives of 4-methyl pyridine-2- amino", J.J. Vora, SB Vasava, KC Parmar, SK Chauhan and SS Sharma, EJ Chem. 6(4), 1205-1210(2009).

[31]. "3D-QSAR COMFA/COMSIA studies on 5- aryl-2,2-dialkyl-4- phenyl -3- 2(H)- furanone derivatives as selective COX -2 inhibitors", Puntaambedkar DS, Girdhar R, Yadav MR, Acta Pharm., 56, 157-174, (2006).

[32]. 32. "Semi-empirical methods based 3D-QSAR studies for some substituted schiff bases of pyridine-2-amine"

[33]. KishorArora, Int J Pharm Bio Sc.,5 (3) : 138 - 148, (2014).
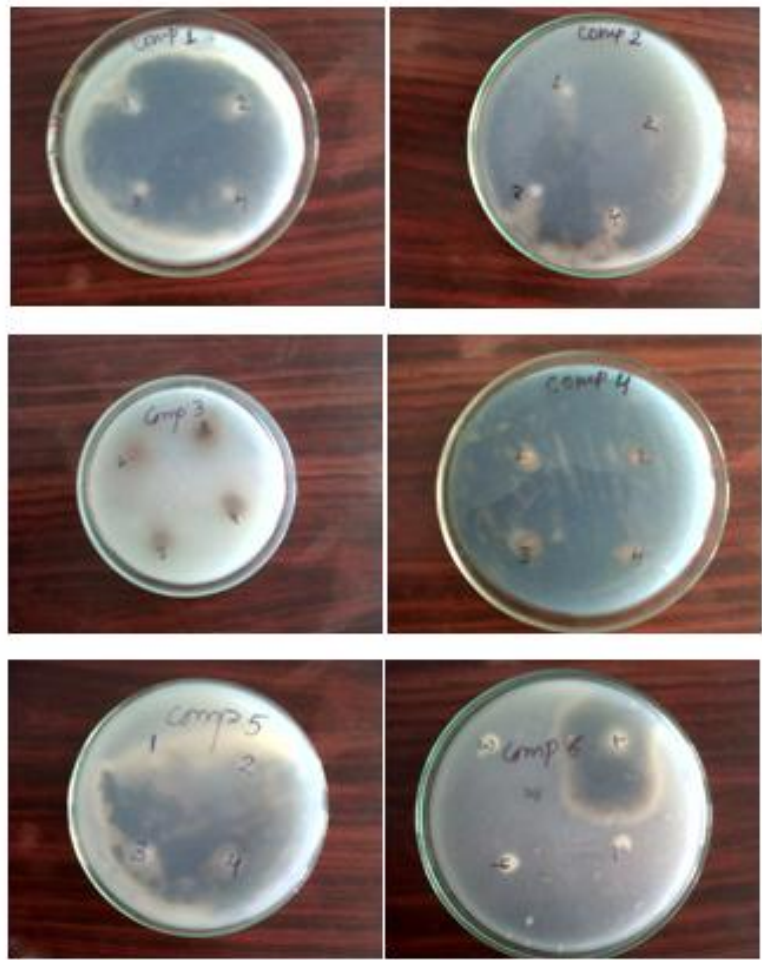

Figure -1: Some notable photographs for anti fungal studies of compounds under study 

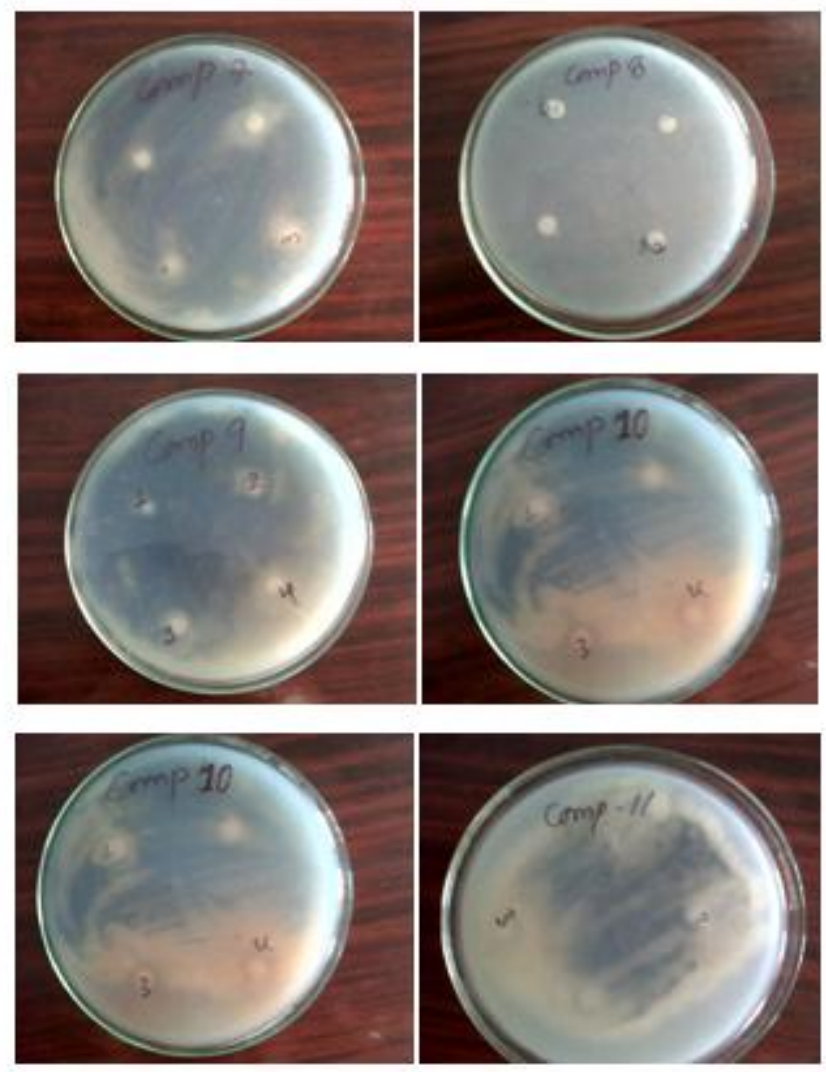

Figure -2: Some notable photographs for anti fungal studies of compounds under study 